\title{
Pluripotential theory for convex bodies in $\mathrm{R}^{N}$
}

\section{Burns ${ }^{1}$, N. Levenberg ${ }^{2, \star}$, S. Ma'u ${ }^{2}$}

1 Department of Mathematics, University of Michigan, Ann Arbor, Michigan 48109-1109, USA

2 Department of Mathematics, University of Auckland, Private Bag 92019, Auckland, New Zealand

Received: 18 March 2004; in final form: 21 June 2004 /

Published online: 21 October 2004 - (C) Springer-Verlag 2004

\section{Introduction}

A plurisubharmonic (psh) function $u$ is maximal on a domain $D \subset \mathbf{C}^{N}$ if, for any relatively compact subdomain $D^{\prime}$, whenever $v$ is psh on $\bar{D}^{\prime}$ and $v \leq u$ on $\partial D^{\prime}$, we have $v \leq u$ in $D^{\prime}$. If $u$ is locally bounded, this is equivalent to $u$ satisfying the homogeneous complex Monge-Ampere equation $\left(d d^{c} u\right)^{N}=0$ in $D$. If $u$ is of class $C^{2}$, then

$$
\left(d d^{c} u\right)^{N}=(2 i \partial \bar{\partial} u)^{N}=4^{N} N ! \operatorname{det}\left[\frac{\partial^{2} u}{\partial z_{j} \partial \bar{z}_{k}}\right]_{j, k=1, \ldots, N} \beta_{N}
$$

where $\beta_{N}=(i / 2)^{N} \prod_{j=1}^{N} d z_{j} \wedge d \bar{z}_{j}$. A result of E. Bedford and M. Kalka [BK] states that if $u \in C^{3}(D)$ and $\left(d d^{c} u\right)^{N-1} \neq 0$, then $D$ can be foliated locally by analytic disks such that the restriction of $u$ to each disk is harmonic. Now let $K \subset \mathbf{C}^{N}$ be compact. The Siciak-Zaharjuta extremal function

$$
V_{K}(z):=\sup \left\{\frac{1}{\operatorname{deg} p} \log |p(z)|: \operatorname{deg} p>0,\|p\|_{K}:=\sup _{z \in K}|p(z)| \leq 1\right\}
$$

(here $p$ is a holomorphic polynomial) is a well-studied object in pluripotential theory. The uppersemicontinuous regularization $V_{K}^{*}$ is either identically $+\infty$ (when $K$ is pluripolar) or it is a locally bounded plurisubharmonic function on $\mathbf{C}^{N}$ which is maximal on $\mathbf{C}^{N} \backslash K$. There are no general techniques to deduce smoothness properties of this function beyond certain criteria for continuity; and explicit computation of $V_{K}^{*}$ and $\left(d d^{c} V_{K}^{*}\right)^{N}$ is virtually impossible. However, let $K \subset \mathbf{R}^{N}$ be a compact, convex body; i.e., $K^{0} \neq \emptyset$, and consider $K$ as a subset of $\mathbf{C}^{N}$. Then, as

\footnotetext{
* Current address: Department of Mathematics, Indiana University, Bloomington,
} Indiana 47405 USA 
shown by Lundin [L1] and later Baran ([Ba1] and [Ba2]), if $K$ is symmetric with respect to the origin; i.e., $K=-K$, then the complement of $K$ in $\mathbf{C}^{N}$ is foliated, in a continuous manner, by one-dimensional analytic disks $L$ (leaves) such that $V_{K}$ restricted to each leaf is harmonic. The main goal of this note is to show that a version of Lundin's result remains valid without the symmetry hypothesis. Indeed, as in the symmetric case, the leaves $L$ are complex ellipses.

Removing the assumption that $K=-K$ is not a mere technical matter; cf., [BCL] where a natural condition that holds for such $K$ - the extremal-like function

$$
V_{K}^{(1)}(z):=\sup \left\{V_{\ell(K)}(\ell(z)): \ell \text { is complex affine }\left(\ell: \mathbf{C}^{N} \rightarrow \mathbf{C}\right)\right\}
$$

coincides with $V_{K}$ - is shown to fail for the standard (nonsymmetric) simplex $S_{2}:=\left\{\left(x_{1}, x_{2}\right) \in \mathbf{R}^{2}: x_{1}, x_{2} \geq 0, x_{1}+x_{2} \leq 1\right\}$ in $\mathbf{R}^{2}$. The assumption that $K=-K$ means that $K$ is the unit ball for a norm on $\mathbf{R}^{N}$; Baran ([Ba1], [Ba2]) exploited this fact and properties of generalized Joukowski maps $t \rightarrow 1 / 2(a t+$ $\bar{b} / t), a, b \in \mathbf{C},|t| \geq 1$, to get explicit formulas for the leaves $L$.

The main idea, conceived by the first author several years ago, is to approximate $K$ from above by a decreasing sequence of compact sets $\left\{K_{j}\right\}$ in $\mathbf{C}^{N}$ of the form $K_{j}=\bar{D}_{j}$, where each $D_{j}$ is a strictly convex domain. For each $D_{j}$ one can apply the Lempert theory (cf., the appendix in [M]) to get analytic disks $L_{j}$ such that $V_{K_{j}}$ restricted to each $L_{j}$ is harmonic. Precisely, in the $d u a l$ set $D_{j}^{\prime}$, the Kobayashi geodesics through a fixed point transform via a nonholomorphic Kelvin-like transform to leaves $L_{j}$ in the complement of $D_{j}$; taking limits as $j \rightarrow \infty$ we obtain our result. We present a self-contained version of this story, modulo proofs of the Lempert results utilized.

The motivation for this research is two-fold: despite the negative results on $S_{2}$ demonstrated in [BCL], such a foliation does, indeed, exist for the simplex. This was known by Baran ([Ba1] and [Ba2]). Moreover, Lundin [L2] himself anticipated such a result; his motivation was to verify the following.

Conjecture 0.1 (Lundin). Let $K \subset \mathbf{R}^{2}$ be a convex body. Then $K$ is not a disk or the region bounded by an ellipse if and only iffor any function $f$ which is harmonic on a simply connected neighborhood $U \neq \mathbf{R}^{2}$ of $K$ but which is not harmonic on all of $\mathbf{R}^{2}$, the greatest geometric degree of convergence for approximation of $f$ by general polynomials is strictly smaller than the greatest geometric degree of convergence for approximation of $f$ by harmonic polynomials.

We will explain the terminology, and verify Lundin's conjecture, in section 3. In the next section we provide background material on extremal plurisubharmonic functions and Lempert theory; and in section 2 we prove our main result, Theorem 2.4 , on the existence of varieties on which $V_{K}$ is harmonic for $K \subset \mathbf{R}^{N}$ a compact, convex body. Finally, in section 4 we describe a second application of our main theorem. We generalize the results of [BCL] in showing that for "most" convex bodies $K \subset \mathbf{R}^{2}, V_{K}^{(1)} \neq V_{K}$. Moreover, we give a geometric description of the set

$$
\left\{z \in \mathbf{C}^{2}: V_{K}^{(1)}(z)<V_{K}(z)\right\}
$$

and show that these sets are large (in particular, unbounded). 
The second author would like to thank many people, including T. Bagby, T. Bloom, E. Bedford, D. Khavinson, S. Pinchuk and B. A. Taylor for valuable discussions.

\section{Background}

The Siciak-Zaharjuta extremal function $V_{K}$ of a compact set $K \subset \mathbf{C}^{N}$ was defined in (0.1). As an example, for $K=B(a, R)$ a closed ball of radius $R$ centered at $a, V_{K}(z)=\log ^{+}(|z-a| / R)$ (cf., p. $185[\mathrm{~K}]$ ). For a convex body $K$ in $\mathbf{R}^{N}$, or a compact convex set $K$ in $\mathbf{C}^{N}$ with nonempty interior (in $\mathbf{C}^{N}$ ), it is known that $V_{K}$ is a uniformly continuous psh function on $\mathbf{C}^{N}$ satisfying $V_{K}=0$ on $K$, $K=\left\{z \in \mathbf{C}^{N}: V_{K}(z)=0\right\}$, and $C_{1}+\log ^{+}|z| \leq V_{K}(z) \leq C_{2}+\log ^{+}|z|$ for constants $C_{1}, C_{2}$ depending on $K$. For future reference, global psh functions $u$ satisfying such an inequality, with constants $C_{1}, C_{2}$ depending on $u$, form the class $L^{+}\left(\mathbf{C}^{N}\right)$; global psh functions $u$ satisfying only the upper bound condition form the class $L\left(\mathbf{C}^{N}\right)$; and compact sets $K$ with $V_{K}$ continuous are called regular. For a bounded set $E \subset \mathbf{C}^{N}$, one defines

$$
V_{E}(z):=\sup \left\{u(z): u \in L\left(\mathbf{C}^{N}\right), u \leq 0 \text { on } E\right\} ;
$$

this coincides with $(0.1)$ when $E$ is compact (Theorem 5.1.7 [K]). Given a bounded domain $D \subset \mathbf{C}^{N}$ and a point $z_{0} \in D$,

$G_{D}\left(z ; z_{0}\right):=\sup \left\{u(z): u \operatorname{psh}\right.$ in $D, u \leq 0, u(z)-\log \left|z-z_{0}\right|=0(1)$ as $\left.z \rightarrow z_{0}\right\}$

is the pluricomplex Green function for $D$ with pole at $z_{0}$; if $z_{0}=0$ we simply write $G_{D}(z)$. For example, for an open ball of radius $R$ centered at $a, G_{D}(z ; a)=$ $\log (|z-a| / R)$.

We make a few remarks on notation:

1. Given a point $z=\left(z_{1}, \ldots, z_{N}\right) \in \mathbf{C}^{N}$, we write, as usual, $\bar{z}=\left(\bar{z}_{1}, \ldots, \bar{z}_{N}\right)$. However, for a set $E \subset \mathbf{C}^{N}$, we use $\bar{E}$ to denote the closure of $E$ and we write $E^{*}$ to denote the conjugate set; i.e.,

$$
E^{*}:=\left\{\bar{z} \in \mathbf{C}^{N}: z \in E\right\} .
$$

2. We use $|\cdot|$ to denote the Euclidean $\left(\ell^{2}\right)$ norm of a vector in $\mathbf{C}^{N}$ for any $N=$ $1,2, \ldots$

3. We will use the notation $\langle a, b\rangle$ to denote the complex bilinear form $<a, b>=\sum_{j=1}^{N} a_{j} b_{j}$ in $\mathbf{C}^{N}$. Note the usual Hermitian inner product is $<a, \bar{b}>=\sum_{j=1}^{N} a_{j} \bar{b}_{j}$.

A bounded domain $D$ in $\mathbf{C}^{N}$ with $C^{2}$-boundary is said to be strictly lineally convex if for each $a \in \partial D$, the complex tangent hyperplane

$$
T_{a}^{\mathbf{C}}(\partial D):=\left\{\zeta \in \mathbf{C}^{N}: \sum_{j=1}^{N}\left(\zeta_{j}-a_{j}\right) \frac{\partial \rho}{\partial z_{j}}(a)=0\right\},
$$


where $\rho \in C^{2}(\bar{D})$ is a defining function for $D$, satisfies $T_{a}^{\mathbf{C}}(\partial D) \cap \bar{D}=\{a\}$. Thus $\operatorname{Hess}_{\rho}(a, w):=2 \Re\left(\sum_{j, k=1, \ldots, N} \frac{\partial^{2} \rho}{\partial z_{j} \partial z_{k}}(a) w_{j} w_{k}\right)+2 \sum_{j, k=1, \ldots, N} \frac{\partial^{2} \rho}{\partial z_{j} \partial \bar{z}_{k}}(a) w_{j} \bar{w}_{k}>0$ for each $a \in \partial D$ and each $w \neq 0$ satisfying $\sum_{j=1}^{N} w_{j} \frac{\partial \rho}{\partial z_{j}}(a)=0$. A stronger notion is that of strict convexity: a bounded domain $D$ in $\mathbf{C}^{N}$ with $C^{2}$-boundary is said to be strictly convex if it has a defining function $\rho$ which satisfies $\operatorname{Hess}_{\rho}(a, w)>0$ for each $a \in \partial D$ and each $w \neq 0$ in the real tangent space to $\partial D$ at $a$; i.e., $w$ satisfies $\mathfrak{R}\left[\sum_{j=1}^{N} w_{j} \frac{\partial \rho}{\partial z_{j}}(a)\right]=0$. For the applications of this paper, it suffices to consider strictly convex sets (and decreasing limits of such sets); however, many of the results remain valid in the strictly lineally convex setting, which is also a more natural framework for the Lempert theory.

We summarize the features of Lempert's works [Le1], [Le2] and [Le3] that we will need. Start with a bounded, strictly lineally convex domain $D$ in $\mathbf{C}^{N}$ containing the origin 0 ; for simplicity we assume that the boundary of $D$ is real-analytic. We define the dual

$$
D^{\prime}:=\left\{z^{\prime} \in \mathbf{C}^{N}:<z, z^{\prime}>\neq 1 \text { for all } z \in \bar{D}\right\} .
$$

This is a bounded domain in $\mathbf{C}^{N}$ containing 0. For example, if $D$ is the open ball of radius $R$ centered at $0, D^{\prime}$ is the open ball of radius $1 / R$ centered at 0 . Note that for each $z^{\prime} \in D^{\prime}$,

$$
H_{z^{\prime}}:=\left\{z \in \mathbf{C}^{N}:<z, z^{\prime}>=1\right\}
$$

is a complex hyperplane with $H_{z^{\prime}} \cap \bar{D}=\emptyset$. Next, define a Kelvin transform from $D^{\prime} \backslash\{0\}$ to $\mathbf{C}^{N} \backslash \bar{D}$ :

$$
\gamma_{G_{D^{\prime}}}\left(z^{\prime}\right):=\frac{\left(\partial G_{D^{\prime}}\left(z^{\prime}\right) / \partial z_{1}^{\prime}, \ldots, \partial G_{D^{\prime}}\left(z^{\prime}\right) / \partial z_{N}^{\prime}\right)}{\sum_{k=1}^{N} z_{k}^{\prime} \partial G_{D^{\prime}}\left(z^{\prime}\right) / \partial z_{k}^{\prime}}
$$

where $G_{D^{\prime}}$ is the pluricomplex Green function for $D^{\prime}$ with pole at 0 . From Lempert's work, $\gamma_{G_{D^{\prime}}}$ is a real-analytic diffeomorphism of $D^{\prime} \backslash\{0\}$ to $\mathbf{C}^{N} \backslash \bar{D}$; and

$$
V_{\bar{D}}\left(\gamma_{G_{D^{\prime}}}\left(z^{\prime}\right)\right)=-G_{D^{\prime}}\left(z^{\prime}\right) \text {. }
$$

In general, Lempert's Kelvin-like transform takes solutions $u$ of a homogeneous complex Monge-Ampere equation $\left(d d^{c} u\right)^{N}=0$ into another solution $\tilde{u}$; i.e., $\left(d d^{c} \tilde{u}\right)^{N}=0$, but this transformation need not preserve plurisubharmonicity: indeed, in our situation, if $u=G_{D^{\prime}}$ (psh) then $\tilde{u}=-V_{\bar{D}}$ (plurisuperharmonic).

The following results hold for $D$ strictly (lineally) convex with real-analytic boundary:

(I) $D^{\prime}$ is strictly lineally convex with real-analytic boundary.

(II) For each $c<0$, the sublevel sets

$$
D_{c}^{\prime}:=\left\{z^{\prime} \in D^{\prime}: G_{D^{\prime}}\left(z^{\prime}\right)<c\right\}
$$

are strictly lineally convex with real-analytic boundary. 
(III) For each $c<0$, the sublevel sets

$$
D_{c}:=\left\{z \in \mathbf{C}^{N}: V_{\bar{D}}<-c\right\}
$$

are strictly (lineally) convex with real-analytic boundary; indeed, $\left(D_{c}\right)^{\prime}=$ $D_{c}^{\prime}$.

For example, if $D$ is the unit ball, so is $D^{\prime}$ and $G_{D^{\prime}}\left(z^{\prime}\right)=\log \left|z^{\prime}\right|$ so that $z=\gamma_{G_{D^{\prime}}}\left(z^{\prime}\right)=\frac{\bar{z}^{\prime}}{\left|z^{\prime}\right|^{2}}$. Thus for $\left|z^{\prime}\right|<1$,

$$
V_{\bar{D}}\left(\gamma_{G_{D^{\prime}}}\left(z^{\prime}\right)\right)=\log ^{+}\left|\gamma_{G_{D^{\prime}}}\left(z^{\prime}\right)\right|=\log ^{+} 1 /\left|z^{\prime}\right|=\log 1 /\left|z^{\prime}\right|=-\log \left|z^{\prime}\right| ;
$$

i.e., $V_{\bar{D}}(z)=-\log \left|z^{\prime}\right|=\log |z|$ for $|z|>1$.

For a geometric description of (1.6), note that if $z^{\prime} \in D^{\prime}, G_{D^{\prime}}\left(z^{\prime}\right)=c<0$, and $z=\gamma_{G_{D^{\prime}}}\left(z^{\prime}\right)$, taking $\rho=G_{D^{\prime}}-c$ and $a=z^{\prime}$ in (1.2) and using (1.5),

$$
T_{z^{\prime}}^{\mathbf{C}}\left(\partial D_{c}^{\prime}\right)=\left\{\zeta \in \mathbf{C}^{N}:<\zeta, z>=1\right\}
$$

Similarly, from (III), the complex hyperplane $H_{z^{\prime}}:=\left\{\zeta \in \mathbf{C}^{N}:<\zeta, z^{\prime}>=1\right\}$, which lies in $\mathbf{C}^{N} \backslash \bar{D}$, coincides with $T_{z}^{\mathbf{C}}\left(\partial D_{c}\right)$. Thus

$$
-G_{D^{\prime}}\left(z^{\prime}\right)=V_{\bar{D}}(z)=\inf _{x \in H_{z^{\prime}}} V_{\bar{D}}(x) .
$$

Conversely, given a complex hyperplane $H$ disjoint from $\bar{D}, H=T_{z}^{\mathrm{C}}\left(\partial D_{c}\right)$ for some $c<0$ and $z \in \partial D_{c}$. Then $z=\gamma_{G_{D^{\prime}}}\left(z^{\prime}\right)$ where $z^{\prime} \in D^{\prime}$ and $H=H_{z^{\prime}}$. Equation (1.7) will be a key to understanding the solution of the Lundin approximation conjecture in section 3 .

\section{Foliations}

Let $\Delta$ denote the open unit disk in $\mathbf{C}$ and let $T:=\partial \Delta$. Given $E \subset \mathbf{C}^{N}$ compact, we let $\kappa_{E}$ denote the set of all $f: \mathbf{C} \backslash \bar{\Delta} \rightarrow \mathbf{C}^{N}$ holomorphic with $|f(t)| /|t|$ bounded; $f$ has a continuous extension to $T$; and $f(T) \subset E$. We recall the following result, due to Lempert (see the appendix in $[\mathrm{M}]$; a nice exposition has also been given by S. Borell [Bo]).

Theorem 2.1. Let $E=\bar{D} \subset \mathbf{C}^{N}$ where $D$ is a bounded, strictly lineally convex domain with real-analytic boundary. There exists a foliation of $\mathbf{C}^{N} \backslash$ E by analytic disks $L=f(\mathbf{C} \backslash \bar{\Delta})$ where $f \in \kappa_{E}$ and $V_{E}(f(t))=\log |t|,|t| \geq 1$.

We sketch the proof of the existence of the analytic disks $L$. Without loss of generality, we may assume $D$ contains the origin. Then

$$
D^{\prime}:=\left\{z^{\prime} \in \mathbf{C}^{N}:<z, z^{\prime}>=\sum_{j=1}^{N} z_{j} z_{j}^{\prime} \neq 1 \text { for all } z \in D\right\}
$$

is a strictly lineally convex domain with real-analytic boundary containing the origin. For a nonzero vector $v$ in $\mathbf{C}^{N}$ and a point $z^{\prime} \in D^{\prime}$, a holomorphic map $g=\left(g_{1}, \ldots, g_{N}\right): \Delta \rightarrow D^{\prime}$ is a Kobayashi geodesic with respect to $z^{\prime} \in D^{\prime}$ and $v$ 
if $g(0)=z^{\prime}, g^{\prime}(0)=\lambda v$ for some $\lambda>0$, and $\lambda$ is maximal among mappings with this property. In the strictly lineally convex setting, Lempert [L3] showed that Kobayashi geodesics through $z^{\prime}=0$ exist, extend to $T$, and foliate $D^{\prime} \backslash\{0\}$. Moreover, on each such leaf, $G(g(s)):=G_{D^{\prime}}(g(s))=\log |s|,|s| \leq 1$. Then for $|t| \geq 1$,

$$
f(t):=\gamma_{G_{D^{\prime}}}(g(1 / t))=\gamma_{G}(g(1 / t))
$$

defines a leaf $L$ of the foliation of $\mathbf{C}^{N} \backslash E$. To see this, we have

$$
V_{E}(z)=-G\left(\gamma_{G}^{-1}(z)\right)
$$

from (1.6). The function $f$ defined in (2.2) is clearly continuous on $\mathbf{C} \backslash \Delta$ with $f(T) \subset E$. Moreover, using (2.3) and (2.2),

$$
\begin{aligned}
V_{E}(f(t)) & =-G\left(\gamma_{G}^{-1}(f(t))\right)=-G\left(\gamma_{G}^{-1}\left(\gamma_{G}(g(1 / t))\right)\right. \\
& =-G((g(1 / t))=-\log |1 / t|=\log |t|
\end{aligned}
$$

for $t \in \mathbf{C} \backslash \Delta$. This shows that $|f(t)| /|t|$ is bounded; for $E \subset B(0, R)$ for some $R$ yields

$$
\log |t|=V_{E}(f(t)) \geq V_{B(0, R)}(f(t)) \geq \log |f(t)|-\log R .
$$

It remains to prove the holomorphicity of $f$. We first show that $\partial G / \partial z_{j}^{\prime} \circ g$ is holomorphic, $j=1, \ldots, N$. To this end, fix $z^{\prime}=g(\alpha), \alpha \in \Delta$. Since $(G \circ g)(s)$ is harmonic for $s \in \Delta$, we have $\partial^{2}(G \circ g)(\alpha) / \partial s \partial \bar{s}=0$. After a complex-linear change of coordinates, we can assume $\left(\partial g_{j}(\alpha) / \partial s\right)_{j=1, \ldots, N}=(1,0, \ldots, 0)$. Then

$$
\partial^{2}(G \circ g)(\alpha) / \partial s \partial \bar{s}=\partial^{2} G\left(z^{\prime}\right) / \partial z_{1}^{\prime} \partial \bar{z}_{1}^{\prime}=0 .
$$

But then from plurisubharmonicity of $G$; i.e., positive semi-definiteness of the complex Hessian $\left[\partial^{2} G\left(z^{\prime}\right) / \partial z_{j}^{\prime} \partial \bar{z}_{k}^{\prime}\right]_{j, k=1, \ldots, N}$, we have

$$
0=\partial^{2} G\left(z^{\prime}\right) / \partial z_{j}^{\prime} \partial \bar{z}_{1}^{\prime}=\partial / \partial \bar{s}\left(\partial G / \partial z_{j}^{\prime} \circ g\right)(\alpha) \text { for } j=1, \ldots, N \text {. }
$$

From the definitions of $\gamma_{G}$ and $f$, it follows immediately that $f$ is holomorphic.

We remark that if $K^{*}=K$, i.e., $z \in K$ if and only if $\bar{z} \in K$, then $V_{K}(z)=$ $V_{K}(\bar{z})$. In particular, this holds if $K \subset \mathbf{R}^{N}$. Similarly, for a bounded domain $U$ containing the origin 0 , if $U^{*}=U$, then $G_{U}(z)=G_{U}(\bar{z})$ where $G_{U}$ is the pluricomplex Green function with pole at 0 . Another important observation is that a general compact, convex body in $\mathbf{R}^{N}$ can be approximated from above by sets fulfilling the hypotheses of Theorem 2.1.

Proposition 2.2. Let $K \subset \mathbf{R}^{N}$ be a compact, convex body. Then there exist $\left\{K_{j}\right\}$ compact, $K_{j}=\bar{D}_{j} \subset \mathbf{C}^{N}$, where $\left\{D_{j}\right\}$ are strictly convex domains having realanalytic boundaries such that $K_{j}^{*}=K_{j}, K_{j+1} \subset K_{j}$ and $K=\cap_{j} K_{j}$.

Under the hypothesis of Proposition 2.2, the Siciak-Zaharjuta extremal functions $V_{K_{j}} \nearrow V_{K}$ uniformly on $\mathbf{C}^{N}$. To see this, note that $V_{K}$ and each $V_{K_{j}}$ are continuous in $\mathbf{C}^{N}$. Thus $V_{K_{j}} \nearrow V_{K}$ uniformly on compact sets in $\mathbf{C}^{N}$ by Dini's theorem. But, since $V_{K_{j}}=0$ on $K_{j}$, $\left\|V_{K_{j}}-V_{K}\right\|_{K_{j}}=\left\|V_{K}\right\|_{K_{j}}$; moreover, $V_{K}-\left\|V_{K}\right\|_{K_{j}} \leq V_{K_{j}}$ from (1.1) so that $V_{K_{j}} \nearrow V_{K}$ uniformly on all of $\mathbf{C}^{N}$. 
Lemma 2.3. Let $E=\bar{D}$ where $D$ is a bounded, strictly lineally convex domain containing the origin and with real-analytic boundary, and let $L(f):=f(\mathbf{C} \backslash \bar{\Delta})$ be the leafassociated to a mapping $f$. If $E^{*}=E$, where $E^{*}:=\left\{z \in \mathbf{C}^{N}: \bar{z} \in E\right\}$, then the function $\tilde{f}(t)=\overline{f(\bar{t})}$ defines a leaf $\tilde{L}=\tilde{L}(\tilde{f})$. In particular, $\tilde{L}(\tilde{f})=(L(f))^{*}$.

Proof. Note that $E^{*}=E$ if and only if $D^{\prime *}=D^{\prime}$ as follows from formula (2.1). Hence $G\left(z^{\prime}\right)=G\left(\overline{z^{\prime}}\right)$ where $G$ is the pluricomplex Green function for $D^{\prime}$ with pole at the origin. Let $L(f)$ be the leaf associated to $f$ given in (2.2) via a Kobayashi geodesic $g$ for a point $z^{\prime} \in D^{\prime}$ and a direction $v$. The function $\tilde{g}(s):=\overline{g(\bar{s})}$ is easily seen to be a Kobayashi geodesic for $\overline{z^{\prime}}$ and $\bar{v}$. The Kelvin transform applied to $\tilde{g}$ gives us the leaf $\tilde{L}=\tilde{L}(\tilde{f})$ where $\tilde{f}(t)=\gamma_{G}(\tilde{g}(1 / t))=\gamma_{G}(\overline{g(1 / \bar{t})})$. To complete the proof, it suffices to show that $\tilde{f}(t)=\overline{f(\bar{t})}$ which follows if

$$
\gamma_{G}\left(\bar{z}^{\prime}\right)=\overline{\gamma_{G}\left(z^{\prime}\right)}
$$

for all $z^{\prime} \in D^{\prime} \backslash\{0\}$. But this is a direct calculation using $G\left(z^{\prime}\right)=G\left(\overline{z^{\prime}}\right)$.

Theorem 2.4. Let $K \subset \mathbf{R}^{N}$ be a convex body. Through any point $q \in \mathbf{C}^{N} \backslash K$ there is a one-dimensional variety $L_{q}=F(\mathbf{C} \backslash \bar{\Delta})$ where $F=\left(F_{1}, \ldots, F_{N}\right)$ with $F_{n}(t)=a_{n 0}+a_{n 1} t+\bar{a}_{n 1} / t$ for some $a_{n 0} \in \mathbf{R}$ and $a_{n 1} \in \mathbf{C} ; F(T) \subset K ;$ and $V_{K}(F(t))=\log |t|$ for $t \in \mathbf{C} \backslash \Delta$. Moreover, $L_{q}^{*}=\tilde{F}(\mathbf{C} \backslash \bar{\Delta})$ is a variety conjugate to $L_{q}$, where $\tilde{F}_{n}(t)=a_{n 0}+\bar{a}_{n 1} t+a_{n 1} / t ; \tilde{F}(T)=F(T)$; and $V_{K}(\tilde{F}(t))=\log |t|$ for $t \in \mathbf{C} \backslash \Delta$.

Note that since $V_{K}(z)=V_{K}(\bar{z})$, we expect to have pairs of conjugate varieties. As an example, let $K=\left\{\left(z_{1}, z_{2}\right) \in \mathbf{C}^{2}: \Im z_{1}=\Im z_{2}=0,\left(\Re z_{1}\right)^{2}+\left(\Re z_{2}\right)^{2} \leq 1\right\}$ be the real unit disk in $\mathbf{R}^{2} \subset \mathbf{C}^{2}$. In this case, the family of leaves

$$
L(c):=f_{c}(\mathbf{C} \backslash \bar{\Delta}) \text { where } f_{c}(t)=\left(\frac{1}{2}\left(c_{1} t+\bar{c}_{1} / t\right), \frac{1}{2}\left(c_{2} t+\bar{c}_{2} / t\right)\right)
$$

for $c=\left(c_{1}, c_{2}\right)$ belonging to the parameter space

$$
\left\{c=\left(c_{1}, c_{2}\right) \in \mathbf{C}^{2}:\left|c_{1}\right|^{2}+\left|c_{2}\right|^{2}+\left|c_{1}^{2}+c_{2}^{2}\right|=2\right\}
$$

(modulo the circle action; i.e., $L(c)=L\left(c^{\prime}\right)$ if and only if $c=e^{i \theta} c^{\prime}$ ), provides a continuously varying foliation of $\mathbf{C}^{2} \backslash K$ (cf., [Ba1]).

Proof of Theorem 2.4. We may assume $0 \in K$. Let $\left\{K_{j}\right\}$ be a sequence of strictly convex sets as in Proposition 2.2. These sets are contained in a fixed ball $B(0, R)$. We claim that the union

$$
\mathcal{F}:=\left\{f \in \cup_{j} \kappa_{K_{j}}: V_{K_{j}}(f(t))=\log |t|,|t| \geq 1 \text {, for some } j\right\}
$$

of all holomorphic mappings $f: \mathbf{C} \backslash \Delta \rightarrow \mathbf{C}^{N}$ which yield a leaf (as in Theorem 2.1 ) for some $K_{j}$ forms a normal family. To see this, first observe that for any $f \in \mathcal{F}$,

$$
|f(t)| /|t| \leq R \text { for all }|t| \geq 1 .
$$


For $K_{j} \subset B(0, R)$ implies that $V_{B(0, R)}(z)=\log ^{+}(|z| / R) \leq V_{K_{j}}(z)$; now apply this inequality to $z=f(t)$ and use $V_{K_{j}}(f(t))=\log |t|$. Thus we may write a component function $f_{n}$ of $f=\left(f_{1}, \ldots, f_{N}\right)$ in a Laurent series expansion about $\infty$ of the form

$$
f_{n}(t)=a_{n 0}+a_{n 1} t+\sum_{k=1}^{\infty} a_{-n k} t^{-k},
$$

and we have from (2.5) that

$$
\left|a_{n 1}\right|=\left|\lim _{t \rightarrow \infty} f_{n}(t) / t\right| \leq \limsup _{t \rightarrow \infty}\left|f_{n}(t)\right| /|t| \leq \limsup _{t \rightarrow \infty}|f(t)| /|t| \leq R .
$$

Now consider the function $g_{n}(s):=a_{n 0}+\sum_{k=1}^{\infty} a_{-n k} s^{k}$. This is holomorphic on $\Delta$ and continuous on $\bar{\Delta}$ and agrees with $f_{n}(1 / s)-a_{n 1} / s$ on $\Delta \backslash\{0\}$. Moreover, on $T$, from the previous estimate and the fact that $f(T) \subset K_{j} \subset B(0, R)$,

$$
\left|g_{n}(s)\right|=\left|f_{n}(1 / s)-a_{n 1} / s\right| \leq 2 R .
$$

Thus we have shown that the family of holomorphic functions $\mathcal{G}$ on the unit disk $\Delta$ defined by

$$
\begin{aligned}
\mathcal{G} & :=\left\{g=\left(g_{1}, \ldots, g_{N}\right): g_{n}(s)\right. \\
& =a_{n 0}+\sum_{k=1}^{\infty} a_{-n k} s^{k}, \text { where } f=\left(f_{1}, \ldots, f_{N}\right) \in \mathcal{F} \text { with } \\
f_{n}(t) & \left.=a_{n 0}+a_{n 1} t+\sum_{k=1}^{\infty} a_{-n k} t^{-k}\right\}
\end{aligned}
$$

is uniformly bounded and hence normal. From this it follows easily that $\mathcal{F}$ is normal.

A sequence $\left\{f^{(j)}\right\} \subset \mathcal{F}$ might converge to a degenerate (constant) mapping $g$; e.g., if $K_{j}=\overline{B(0,1 / j)}$ and $f^{(j)}(t)=(t / j, 0, \ldots, 0)$, then $f^{(j)} \rightarrow g$ where $g(t)=$ $(0,0, \ldots, 0)$. We need to avoid degenerate limits in our situation. Fix $q \in \mathbf{C}^{N} \backslash K$; then $q \notin K_{j}$ for $j \geq j_{0}=j_{0}(q)$. We show there exist $M=M(q)<+\infty$ and $\epsilon=\epsilon(q)>0$ such that for all $j \geq j_{0}$ there exists $f_{j} \in \kappa_{K_{j}}$, i.e., $f_{j}: \mathbf{C} \backslash \Delta \rightarrow \mathbf{C}^{N}$ with $V_{K_{j}}\left(f_{j}(t)\right)=\log |t|,|t| \geq 1$ and

$$
f_{j}\left(t_{j}\right)=q \text { for some } t_{j} \text { satisfying } 1+\epsilon<\left|t_{j}\right| \leq M .
$$

To see this, we simply note that $V_{K} \in L\left(\mathbf{C}^{N}\right)$ implies

$$
V_{K_{j}}(q)=V_{K_{j}}\left(f_{j}\left(t_{j}\right)\right)=\log \left|t_{j}\right| \leq V_{K}(q) \leq C+\log |q|=: \log M ;
$$

and, for $j \geq j_{0}$,

$$
V_{K_{j}}(q)=V_{K_{j}}\left(f_{j}\left(t_{j}\right)\right)=\log \left|t_{j}\right| \geq V_{K_{j_{0}}}(q)=: \log (1+\epsilon)>0
$$

since $q \notin K_{j_{0}}$.

For each $j \geq j_{0}$, we now pick a map $f_{j} \in \kappa_{K_{j}}$ with $f_{j}\left(t_{j}\right)=q$ for some $t_{j}$ with $1+\epsilon \leq\left|t_{j}\right| \leq M$. Next, we take a subsequence, which we again call $\left\{f_{j}\right\}$, with the property that $t_{j} \rightarrow t_{0}$ for some $t_{0}$. Note that since $f_{j}(t) \subset K_{j}$ for $|t|=1$ 
and each $f_{j}$ is unbounded, any normal limit of these maps with $f_{j}\left(t_{j}\right)=q$ for some $1+\epsilon \leq\left|t_{j}\right| \leq M$ must be nonconstant. Since each component function $f_{j n}$ of $f_{j}=\left(f_{j 1}, \ldots, f_{j N}\right)$ has a Laurent series expansion of the form

$$
f_{j n}(t)=a_{j n 0}+a_{j n 1} t+\sum_{k=1}^{\infty} a_{-j n k} t^{-k}
$$

where $\left|a_{j n 1}\right| \leq R$, by taking a further subsequence, we can assume that the sequence of coefficients $\left\{a_{j n 1}\right\}_{j=1,2, \ldots}$ converges to $a_{n 1}$ for $n=1,2, \ldots, N$. This subsequence of maps lies in $\mathcal{F}$; thus we may choose a subsequence converging normally to $F: \mathbf{C} \backslash \bar{\Delta} \rightarrow \mathbf{C}^{N}$ with $F\left(t_{0}\right)=q$ for some $t_{0}$ with $1+\epsilon \leq\left|t_{0}\right| \leq M$ and so that each component function $F_{n}$ of $F=\left(F_{1}, \ldots, F_{N}\right)$ has a Laurent series expansion of the form

$$
F_{n}(t)=a_{n 0}+a_{n 1} t+\sum_{k=1}^{\infty} a_{-n k} t^{-k} .
$$

We will soon see that $F$ is unbounded; i.e., at least one of the coefficients $a_{11}, \ldots, a_{N 1}$ is nonzero.

Now $V_{K_{j}}\left(f_{j}(t)\right)=\log |t|$ for $t \in \mathbf{C} \backslash \Delta$ and $f_{j}$ converges locally uniformly to $F$ on $\mathbf{C} \backslash \Delta$. Since $V_{K_{j}}$ increase monotonically and uniformly to $V_{K}$ on all of $\mathbf{C}^{N}$, we have $V_{K}(F(t))=\log |t|$ for $t \in \mathbf{C} \backslash \bar{\Delta}$. In particular, for $\epsilon>0$ sufficiently small,

$$
V_{K}(F(t)) \leq \epsilon \text { if } 1<|t| \leq 1+\epsilon .
$$

Since $V_{K}$ is uniformly continuous in $\mathbf{C}^{N}$ and $K=\left\{z \in \mathbf{C}^{N}: V_{K}(z)=0\right\} \subset \mathbf{R}^{N}$, it follows that for each component function $F_{n}$ we have $\lim _{|t| \rightarrow 1^{+}} \mathfrak{\Im} F_{n}(t)=0$. By the reflection principle, we get a holomorphic extension of $F_{n}$ to $\mathbf{C} \backslash\{0\}$ via $\overline{F_{n}(1 / \bar{t})}$ for $0<|t|<1$.

Applying Lemma 2.3 to $K_{j}$ and $f_{j}$, the subsequence $\left\{\tilde{f}_{j}\right\}$, where $\tilde{f}_{j}(t)=\overline{f_{j}(\bar{t})}$, converges normally on $\mathbf{C} \backslash \bar{\Delta}$ to a holomorphic $\tilde{F}=\left(\tilde{F}_{1}, \ldots, \tilde{F}_{N}\right)$ with component functions $\tilde{F}_{n}(t)=\overline{F_{n}(\bar{t})}$ having Laurent series expansions about $\infty$ of the form

$$
\tilde{F}_{n}(t)=\bar{a}_{n 0}+\bar{a}_{n 1} t+\sum_{k=1}^{\infty} \bar{a}_{-n k} t^{-k}
$$

Thus we have shown that

$$
H(t):=\left\{\begin{array}{ll}
F(t), & t \notin \Delta ; \\
\tilde{F}(1 / t), & t \in \Delta \backslash\{0\}
\end{array}= \begin{cases}F(t), & t \notin \Delta ; \\
F(1 / \bar{t}), & t \in \Delta \backslash\{0\}\end{cases}\right.
$$

defines a holomorphic mapping of $\mathbf{C} \backslash\{0\}$ into $\mathbf{C}^{N}$. Now from (2.6) the Laurent series expansion of the $n$-th component of $F$ is of the form

$$
F_{n}(t)=a_{n 0}+a_{n 1} t+\sum_{k=1}^{\infty} a_{-n k} t^{-k}
$$


and from (2.7) the Laurent series expansion of the $n$-th component of $\tilde{F}$ is of the form

$$
\tilde{F}_{n}(t)=\bar{a}_{n 0}+\bar{a}_{n 1} t+\sum_{k=1}^{\infty} \bar{a}_{-n k} t^{-k} .
$$

From (2.8), we have $F\left(e^{i \theta}\right)=\tilde{F}\left(e^{-i \theta}\right)$ for $t=e^{i \theta} \in T$. This gives

$$
a_{n 0}+a_{n 1} e^{i \theta}+\sum_{k=1}^{\infty} a_{-n k} e^{-i k \theta}=\bar{a}_{n 0}+\bar{a}_{n 1} e^{-i \theta}+\sum_{k=1}^{\infty} \bar{a}_{-n k} e^{i k \theta} .
$$

Hence $a_{-n k}=0$ for $k=2,3, \ldots ; a_{n 0}=\bar{a}_{n 0}$; and $a_{-n 1}=\bar{a}_{n 1}$; thus

$$
F_{n}(t)=a_{n 0}+\left(a_{n 1} t+\bar{a}_{n 1} / t\right)
$$

where $a_{n 0}$ must be real (thus $F(T) \subset K \subset \mathbf{R}^{N}$ ). Moreover, we see that at least one of the coefficients $a_{11}, \ldots, a_{N 1}$ is nonzero or else $F$ is constant, contradicting our earlier result. This completes the proof.

Note that the holomorphic map $H(t)$ in (2.8) is of the form

$$
H(t)=a+b t+\bar{b} / t, t \in \mathbf{C} \backslash\{0\}, a \in \mathbf{R}^{N} \text {, and } H(\mathbf{C} \backslash\{0\})=L_{q} \cup L_{q}^{*} \text {. }
$$

We have not verified that one can obtain a foliation of $\mathbf{C}^{N} \backslash K$ in Theorem 2.4. For the applications in the next sections, we only require the existence, through each point $q \in \mathbf{C}^{N} \backslash K$, of a one-dimensional variety $L_{q}=F(\mathbf{C} \backslash \bar{\Delta})$ on which $V_{K}$ is harmonic, as well as the existence of a conjugate leaf $L_{q}^{*}=\tilde{F}(\mathbf{C} \backslash \bar{\Delta})$ with $F(T)=\tilde{F}(T)$.

\section{Approximation}

In this section, which follows closely the presentation in Lundin's thesis [L2], we verify Conjecture 0.1 in Theorem 3.1. To explain the conjecture, let $P_{n}$ denote the (real) vector space of real-valued polynomials in $\mathbf{R}^{N}, N \geq 2$, of degree at most $n$ and let $H_{n} \subset P_{n}$ denote the (real) vector subspace of real-valued harmonic polynomials in $\mathbf{R}^{N}$ of degree at most $n$. For $f$ a real-valued continuous function on $K$, let

$$
R_{n}(f):=\inf \left\{\left\|f-p_{n}\right\|_{K}: p_{n} \in P_{n}\right\}
$$

and

$$
\rho_{n}(f):=\inf \left\{\left\|f-h_{n}\right\|_{K}: h_{n} \in H_{n}\right\} .
$$

Since $H_{n} \subset P_{n}$, clearly $\rho_{n}(f) \geq R_{n}(f)$. Then

$$
R(f):=\limsup _{n \rightarrow \infty} R_{n}(f)^{1 / n}
$$

is called the greatest geometric degree of convergence for approximation of $f$ by general polynomials while

$$
\rho(f):=\limsup _{n \rightarrow \infty} \rho_{n}(f)^{1 / n}
$$

is called the greatest geometric degree of convergence for approximation of $f$ by harmonic polynomials; clearly $\rho(f) \geq R(f)$ for any $f$. 
Theorem 3.1. Let $K \subset \mathbf{R}^{2}$ be a convex body. Then $K$ is not a disk or the region bounded by an ellipse if and only if for any function $f$ which is harmonic on a simply connected neighborhood $U \neq \mathbf{R}^{2}$ of $K$ but which is not harmonic on all of $\mathbf{R}^{2}$, we have $\rho(f)>R(f)$.

Note that if $f$ is harmonic on $\mathbf{R}^{N}$, then for any $K \subset \mathbf{R}^{N}, \rho(f)=R(f)=0$. If $K \subset \mathbf{R}^{N}$ is the region bounded by an ellipsoid $E$; i.e., if $\partial K=E:=\{x \in$ $\left.\mathbf{R}^{N}: Q(x)=0\right\}$ for a quadratic polynomial $Q$ whose degree two homogeneous terms define a positive definite quadratic form, then, as pointed out to us by D. Khavinson, if $p_{n} \in P_{n}$ is a polynomial of degree at most $n$ in $N$ variables we can find a harmonic polynomial $h_{n} \in H_{n}$ of degree at most $n$ that coincides with $p_{n}$ on $E$. Then for any $f$ harmonic on $K$,

$$
\left\|f-p_{n}\right\|_{K} \geq\left\|f-p_{n}\right\|_{E}=\left\|f-h_{n}\right\|_{E}=\left\|f-h_{n}\right\|_{K}
$$

the last equality following from the maximum principle for harmonic functions. Thus $R_{n}(f)=\rho_{n}(f)$ and hence $R(f)=\rho(f)$ for all such $f$; in particular, for $N=2$, we have proved the "if" direction of Theorem 3.1. The proof of the italicised statement, as kindly communicated to us by Khavinson, runs as follows: for each $n$ define a linear operator $T_{n}$ taking the space $P_{n}$ of polynomials of degree at most $n$ into itself via $T_{n}(p):=\Delta(Q p)$ (here $\Delta$ is the Laplacian). Note that $T_{n}: P_{n} \rightarrow P_{n}$ is one-to-one: if $T_{n}(p)=0$, then $\Delta(Q p)=0$ so that $Q p$ is harmonic; however, $Q p=0$ on $E=\partial K$ so by the maximum principle $Q p=0$ on $K$ and $p=0$. In particular, $T_{n-2}$ is surjective so that given $p_{n} \in P_{n}$, we can find $q_{n-2} \in P_{n-2}$ with $T_{n}\left(q_{n-2}\right)=\Delta\left(Q q_{n-2}\right)=\Delta p_{n}$. Then $\Delta\left(Q q_{n-2}-p_{n}\right)=0$ so that $p_{n}-Q q_{n-2}$ is a harmonic polynomial of degree $n$ which agrees with $p_{n}$ on $E=\partial K$.

For the rest of the section, $K$ will be a convex body in $\mathbf{R}^{2}$. It will be convenient to embed $\mathbf{R}^{2}$ into $\mathbf{C}^{2}$ in two different ways. The standard way is to consider

$$
\mathbf{R}^{2}=\left\{\left(z_{1}, z_{2}\right) \in \mathbf{C}^{2}: \Im z_{1}=\Im z_{2}=0\right\}
$$

as usual, we write $z_{1}=x_{1}+i y_{1}$ and $z_{2}=x_{2}+i y_{2}$. Thus $\mathbf{R}^{2}$ is identified with $\mathbf{C}$ via $s:=x_{1}+i x_{2}$. On the other hand, we also define $\left(w_{1}, w_{2}\right)$ coordinates via

$$
w_{1}=z_{1}+i z_{2}, w_{2}=z_{1}-i z_{2} .
$$

In these coordinates, we identify $\mathbf{R}^{2}$ with the image of the embedding of $\mathbf{C}$ into $\mathbf{C}^{2}$ given by $s \rightarrow(s, \bar{s})$; thus

$$
\mathbf{R}^{2}=\left\{\left(w_{1}, w_{2}\right) \in \mathbf{C}^{2}: w_{1}=\bar{w}_{2}\right\}
$$

Given a convex body $K \subset \mathbf{R}^{2}$, we will

(i) utilize the classical Green function $g_{K}\left(x_{1}+i x_{2}\right)$ for $K$ when we consider $K \subset \mathbf{R}^{2}=\mathbf{C}$; i.e., if $s=x_{1}+i x_{2}$, then $V_{K}(s)=g_{K}\left(x_{1}+i x_{2}\right)$ in $(0.1)$;

(ii) utilize the Siciak-Zaharjuta extremal function $V_{K}\left(z_{1}, z_{2}\right)$ for $K$ when we consider $K \subset \mathbf{R}^{2} \subset \mathbf{C}^{2}$. 
In this second case, since $\left(z_{1}, z_{2}\right) \rightarrow S\left(z_{1}, z_{2}\right)=\left(w_{1}, w_{2}\right)=\left(z_{1}+i z_{2}, z_{1}-i z_{2}\right)$ is an invertible complex-linear map, it is easy to see that

$$
\begin{aligned}
V_{\widetilde{K}}\left(w_{1}, w_{2}\right) & =V_{K}\left(z_{1}, z_{2}\right) \text { where } \widetilde{K}:=S(K) \\
& =\left\{\left(w_{1}, w_{2}\right): w_{1} \in K, w_{2}=\bar{w}_{1}\right\}
\end{aligned}
$$

(or use Theorem 5.3.1 of $[\mathrm{K}]$ ).

Now suppose a real-valued $f$ is harmonic on a simply connected neighborhood $U=U(f) \neq \mathbf{R}^{2}$ of $K$ but $f$ is not harmonic on all of $\mathbf{R}^{2}$. Let $\rho:=\rho(f)$ and $R:=R(f)$; then $R \leq \rho$. Let $g_{K}\left(x_{1}+i x_{2}\right)$ be the classical Green function for $K$ as in (i). By a Bernstein-Walsh type theorem for harmonic functions (cf., [ND] or [W]), it follows that $f$ can be extended to a harmonic function on

$$
D_{1 / \rho}:=\left\{\left(x_{1}, x_{2}\right) \in \mathbf{R}^{2}: g_{K}\left(x_{1}+i x_{2}\right)<\log 1 / \rho\right\} ;
$$

the hypothesis on $f$ in Theorem 3.1 means simply that $0<\rho<1$. On the other hand, since $R<1$, by the Bernstein-Walsh theorem for holomorphic functions (cf., $[\mathrm{S}]), f$ can be extended to a holomorphic function of $\left(w_{1}, w_{2}\right)$ on

$$
E_{1 / R}:=\left\{\left(w_{1}, w_{2}\right) \in \mathbf{C}^{2}: V_{\widetilde{K}}\left(w_{1}, w_{2}\right)<\log 1 / R\right\}
$$

(here we are considering real polynomials in $\left(x_{1}, x_{2}\right)$ to be holomorphic polynomials in $\left(w_{1}, w_{2}\right)$ restricted to $\left.w_{1}=\bar{w}_{2}\right)$. Note we always consider holomorphic extensions to a subset of (some) $\mathbf{C}^{2}$ and harmonic extensions to a subset of $\mathbf{R}^{2}$. In order to compare these two types of extension of $f$, we need a lemma.

Lemma 3.2. Let $D$ be a domain in $\mathbf{C}^{2}$ that has a non-empty intersection $U$ with $\mathbf{R}^{2}=\left\{\left(w_{1}, w_{2}\right) \in \mathbf{C}^{2}: w_{1}=\bar{w}_{2}\right\}$. If $F$ is holomorphic in $D$ and the restriction $f$ of $F$ to $U$ is harmonic, then locally in the $\left(w_{1}, w_{2}\right)$ coordinates, $F$ is of the form

$$
F\left(w_{1}, w_{2}\right)=f_{1}\left(w_{1}\right)+f_{2}\left(w_{2}\right)
$$

where $f_{1}, f_{2}$ are holomorphic functions. Moreover, if $f$ is real-valued on $U$, then

$$
F\left(w_{1}, w_{2}\right)=f_{1}\left(w_{1}\right)+\tilde{f}_{1}\left(w_{2}\right)
$$

near $U$ where $\tilde{f}_{1}(t)=\overline{f_{1}(\bar{t})}$.

Proof. Note that (3.2) is equivalent (locally) to $\frac{\partial^{2} F\left(w_{1}, w_{2}\right)}{\partial w_{1} \partial w_{2}}=0$. On $U \subset \mathbf{R}^{2}$ we have $w_{1}=\bar{w}_{2}$; moreover, $f$ is harmonic so

$$
0=\frac{\partial^{2} f\left(w_{1}, \bar{w}_{1}\right)}{\partial w_{1} \partial \bar{w}_{1}}=\frac{\partial^{2} F\left(w_{1}, w_{2}\right)}{\partial w_{1} \partial w_{2}}
$$

for $\left(w_{1}, w_{2}\right) \in U$. However, the function $\frac{\partial^{2} F\left(w_{1}, w_{2}\right)}{\partial w_{1} \partial w_{2}}$ is a holomorphic function on $D$; since it vanishes on $U$, it must vanish identically, proving (3.2). Equation (3.3) is a direct calculation from (3.2) and the assumption that $f$ is real-valued on $U$. 
Consider now, for $r<1$, the sets

$$
\begin{aligned}
\Omega_{1 / r} & :=\left\{\left(w_{1}, w_{2}\right) \in \mathbf{C}^{2}: g_{K}\left(w_{1}\right)<\log 1 / r, g_{K}\left(\bar{w}_{2}\right)<\log 1 / r\right\} \\
& =D_{1 / r} \times D_{1 / r}^{*},
\end{aligned}
$$

which are also simply connected. Then by Lemma 3.2 (equation (3.3)) and the fact that the coordinate projections $\pi_{j}$ onto the $w_{j}$-plane satisfy $\pi_{1}\left(\Omega_{1 / \rho}\right)=D_{1 / \rho}$ and $\pi_{2}\left(\Omega_{1 / \rho}\right)^{*}=D_{1 / \rho}$, we see that $f$ has a holomorphic extension of the form $F\left(w_{1}, w_{2}\right)=f_{1}\left(w_{1}\right)+\tilde{f}_{1}\left(w_{2}\right)$ to the set $\Omega_{1 / \rho}$. The singularities of $F$ are of the form $w_{1}=$ const. or $w_{2}=$ const.; hence $F$ has a singularity on $\partial \Omega_{1 / \rho}$. This means that $f_{1}$ has a singularity on $\partial D_{1 / \rho}$. On the other hand, $f$ can be extended to a holomorphic function $F\left(w_{1}, w_{2}\right)$ on

$$
E_{1 / R}:=\left\{\left(w_{1}, w_{2}\right) \in \mathbf{C}^{2}: V_{\widetilde{K}}\left(w_{1}, w_{2}\right)<\log 1 / R\right\}
$$

but to no larger level set $E_{1 / R^{\prime}}$ for $R^{\prime}<R$. In particular,

$$
\text { if } r<1 \text { is such that } E_{1 / r} \subset \Omega_{1 / \rho} \text {, then } r \geq R \text {. }
$$

Example. Take $K=\bar{\Delta}$ and let

$$
f\left(x_{1}, x_{2}\right)=f\left(x_{1}+i x_{2}\right)=f(s)=\Re\left(\frac{1}{s-2}\right)=\frac{x_{1}-2}{\left(x_{1}-2\right)^{2}+x_{2}^{2}} .
$$

Then $f$ is harmonic in $D_{1 / \rho}=\{s:|s|<2\}(\rho=1 / 2)$ and has a singularity on $\partial D_{1 / \rho}$. The function

$$
F\left(w_{1}, w_{2}\right):=\frac{1}{2}\left(\frac{1}{w_{1}-2}+\frac{1}{w_{2}-2}\right)=f_{1}\left(w_{1}\right)+\tilde{f}_{1}\left(w_{2}\right)
$$

is a holomorphic extension of $f$ where $f_{1}\left(w_{1}\right)=\frac{1}{2}\left(\frac{1}{w_{1}-2}\right)$. Note $F$ is holomorphic on

$$
\begin{aligned}
\left\{\left(w_{1}, w_{2}\right) \in \mathbf{C}^{2}:\left|w_{1}\right|<2,\left|w_{2}\right|<2\right\} & =\left\{\left(w_{1}, w_{2}\right) \in \mathbf{C}^{2}:\left|w_{1}\right|<2,\left|\bar{w}_{2}\right|<2\right\} \\
& =\Omega_{1 / \rho}
\end{aligned}
$$

and has singularities on $\partial \Omega_{1 / \rho}$.

To construct a link between the sets $E_{1 / R}$ and $\Omega_{1 / \rho}$, we define the function

$$
h_{K}(s):=\inf _{w_{2}} V_{\widetilde{K}}\left(s, w_{2}\right) .
$$

From the definition of $h_{K}, V_{\widetilde{K}}\left(w_{1}, w_{2}\right) \geq h_{K}\left(w_{1}\right)$. For $K \subset \mathbf{R}^{2}, V_{K}\left(z_{1}, z_{2}\right)=$ $V_{K}\left(\bar{z}_{1}, \bar{z}_{2}\right)$; in the $\left(w_{1}, w_{2}\right)$ coordinates, this becomes

$$
V_{\widetilde{K}}\left(w_{1}, w_{2}\right)=V_{\widetilde{K}}\left(\bar{w}_{2}, \bar{w}_{1}\right) .
$$

From (3.5) and (3.6), $h_{K}\left(\bar{w}_{2}\right) \leq V_{\widetilde{K}}\left(\bar{w}_{2}, \bar{w}_{1}\right)=V_{\widetilde{K}}\left(w_{1}, w_{2}\right)$ so that

$$
V_{\widetilde{K}}\left(w_{1}, w_{2}\right) \geq \max \left[h_{K}\left(w_{1}\right), h_{K}\left(\bar{w}_{2}\right)\right] .
$$

To compare $g_{K}$ and $h_{K}$, we first prove the following. 
Lemma 3.3. Let $K \subset \mathbf{R}^{2}$ be a convex body. Then $h_{K}(s):=\inf _{w_{2}} V_{\widetilde{K}}\left(s, w_{2}\right)$ is superharmonic in $\mathbf{R}^{2} \backslash K \equiv \mathbf{C} \backslash K$.

Proof. To prove the superharmonicity of $h_{K}$, we revert to the notation in section 1 and summarize the discussion there. Given a strictly convex domain $D \subset \mathbf{C}^{N}$ containing the origin and with real-analytic boundary, $D^{\prime}$ is a strictly convex domain with real-analytic boundary containing the origin 0 , and $G=G_{D^{\prime}}$, the pluricomplex Green function for $D^{\prime}$ with logarithmic pole at 0 , is real-analytic in $\bar{D}^{\prime} \backslash\{0\}$. Given $z^{\prime} \in D^{\prime}$, the complex hyperplane $H_{z^{\prime}}:=\left\{\zeta \in \mathbf{C}^{N}:<\zeta, z^{\prime}>=1\right\}$ lies in $\mathbf{C}^{N} \backslash \bar{D}$ and

$$
-G\left(z^{\prime}\right)=\inf _{x \in H_{z^{\prime}}} V_{\bar{D}}(x) .
$$

Now as $z^{\prime}$ varies over any analytic disk $\delta^{\prime}$ in $D^{\prime}$, since $-G$ is plurisuperharmonic, it follows that $-\left.G\right|_{\delta^{\prime}}$ is superharmonic. From (1.7), the function $w \rightarrow \inf _{x \in H_{w}} V_{\bar{D}}(x)$ is superharmonic for $w \in \delta^{\prime}$. This remains true for $\bar{D}$ replaced by a convex body $K \subset \mathbf{R}^{N}$ as can be seen utilizing a limiting argument and the approximation result in Proposition 2.2.

We work in $\mathbf{R}^{2}$ where we consider $\mathbf{R}^{2}$ as the image of the embedding of $\mathbf{C}$ into $\mathbf{C}^{2}$ given by $s \rightarrow(s, \bar{s})$. Then a convex body $K \subset \mathbf{R}^{2}$, which we may assume contains the origin, can be considered as sitting inside the totally real $2-$ plane $\left\{\left(w_{1}, w_{2}\right): w_{1}=\bar{w}_{2}\right\}$ as the set $\widetilde{K}:=S(K)$ (see (3.1)). Now the hyperplane $H^{s}:=\left\{\left(s, w_{2}\right): w_{2} \in \mathbf{C}\right\}$ is disjoint from $\widetilde{K}$ provided $(s, \bar{s}) \notin \widetilde{K}$. Approximating $K$ from above by $\left\{K_{j}=\bar{D}_{j}\right\}$ as in Proposition 2.2, and writing $\widetilde{K}_{j}=S\left(K_{j}\right), \tilde{D}_{j}=S\left(D_{j}\right)$ - note $S\left(D_{j}\right)$ is strictly convex and contains the origin - if $(s, \bar{s}) \in \mathbf{C}^{2} \backslash \widetilde{K}$, then $(s, \bar{s}) \in \mathbf{C}^{2} \backslash \widetilde{K}_{j}$ for sufficiently large $j$. For such $j$, the family of hyperplanes $\left\{H^{s}\right\}$ as $(s, \bar{s})$ ranges over points in $\mathbf{C}^{2} \backslash \widetilde{K}_{j}$ can be written as hyperplanes $\left\{H_{w^{\prime}}\right\}$ with $w^{\prime}=\left(w_{1}^{\prime}, w_{2}^{\prime}\right) \in\left(\tilde{D}_{j}\right)^{\prime}$ (see (1.4)):

$$
\begin{aligned}
H_{w^{\prime}}: & =\left\{\left(w_{1}, w_{2}\right) \in \mathbf{C}^{2}:<w, w^{\prime}>=w_{1} w_{1}^{\prime}+w_{2} w_{2}^{\prime}=1\right\} \\
& =\left\{\left(s, w_{2}\right): s w_{1}^{\prime}+w_{2} w_{2}^{\prime}=1\right\} .
\end{aligned}
$$

In particular, since $(s, 0) \in H^{s}$ we have $s w_{1}^{\prime}=1$ so that $w_{1}^{\prime}=1 / s$. But then $w_{2}^{\prime}=0$; i.e., $\left(w_{1}^{\prime}, w_{2}^{\prime}\right)=(1 / s, 0)$. This says that the points on the complex line $w_{2}^{\prime}=0$ in $\left(\tilde{D}_{j}\right)^{\prime}$ correspond to the "parallel hyperplanes" $H^{s}=H_{(1 / s, 0)}$ in $\mathbf{C}^{2} \backslash \widetilde{K}_{j}$. Thus

$$
h_{K_{j}}(s):=\inf _{\left(s, w_{2}\right) \in H^{s}} V_{\widetilde{K}_{j}}\left(s, w_{2}\right)=\inf _{x \in H_{(1 / s, 0)}} V_{\widetilde{K}_{j}}(x)=-G_{\left(\tilde{D}_{j}\right)^{\prime}}(1 / s, 0)
$$

for sufficiently large $j$. The functions $h_{K_{j}}(s)=-G_{\left(\tilde{D}_{j}\right)^{\prime}}(1 / s, 0)$ form an increasing sequence of superharmonic functions on a sequence of domains increasing to $\mathbf{C} \backslash K$. We have $V_{\widetilde{K}_{j}} \nearrow V_{\widetilde{K}}$ uniformly on all of $\mathbf{C}^{2}$; in particular, on each hyperplane $H^{s}$ in $\mathbf{C}^{2} \backslash \widetilde{K}$. Thus $h_{K_{j}} \nearrow h_{K}$ on $\mathbf{C} \backslash K$, and on this set $h_{K}$ is superharmonic.

Since $h_{K}(s)=0$ for $s \in K$ and $h_{K}(s)-\log |s|=0(1)$ as $|s| \rightarrow \infty$, we have $g_{K} \leq h_{K}$ and hence

$$
V_{\widetilde{K}}\left(w_{1}, w_{2}\right) \geq \max \left[h_{K}\left(w_{1}\right), h_{K}\left(\bar{w}_{2}\right)\right] \geq \max \left[g_{K}\left(w_{1}\right), g_{K}\left(\bar{w}_{2}\right)\right] .
$$


In terms of sublevel sets, (3.7) says, for any $r<1$,

$$
\begin{aligned}
E_{1 / r} & \subset\left\{\left(w_{1}, w_{2}\right) \in \mathbf{C}^{2}: h_{K}\left(w_{1}\right)<\log 1 / r, h_{K}\left(\bar{w}_{2}\right)<\log 1 / r\right\} \\
& \subset \Omega_{1 / r}=D_{1 / r} \times D_{1 / r}^{*} .
\end{aligned}
$$

Thus we want to decrease $r$ until $E_{1 / r}$ hits a singularity of $F$; from (3.8), the corresponding level set of $h_{K}$ will also hit this singularity.

Proposition 3.4. Let $K \subset \mathbf{R}^{2}$ be a convex body and let $f$ be harmonic in a simply connected neighborhood of $K$ but not on all of $\mathbf{R}^{2}$. We have $g_{K}<h_{K}$ at all points in $\mathbf{R}^{2} \backslash K$ if and only if $R<\rho$.

Proof. Since $g_{K}$ is harmonic on $\mathbf{R}^{2} \backslash K$ and $h_{K}$ is superharmonic on $\mathbf{R}^{2} \backslash K$ with $g_{K} \leq h_{K}$, we have strict inequality on $\mathbf{R}^{2} \backslash K$ if strict inequality holds at one such point. Suppose first that $g_{K}<h_{K}$ on $\mathbf{R}^{2} \backslash K$. Then at each point $\left(w_{1}, w_{2}\right)$ on $\partial \Omega_{1 / \rho}$ we have $h_{K}\left(w_{1}\right)>\log 1 / \rho$ or $h_{K}\left(\bar{w}_{2}\right)>\log 1 / \rho$ so that, from (3.7), $V_{\widetilde{K}}\left(w_{1}, w_{2}\right)>\log 1 / r>\log 1 / \rho$ for some $r<\rho$. Thus $E_{1 / r} \subset \Omega_{1 / \rho}$ so that, from (3.4), $\rho>r \geq R$.

Now suppose $g_{K}=h_{K}$ on $\mathbf{R}^{2} \backslash K$. Take $s$ with $g_{K}(s)=\log 1 / \rho$ at which $f_{1}$ has a singularity. Then

$$
h_{K}(s)=\log 1 / \rho=\inf _{w_{2}} V_{\widetilde{K}}\left(s, w_{2}\right)
$$

so that

$$
\bar{E}_{1 / \rho}=\left\{\left(w_{1}, w_{2}\right) \in \mathbf{C}^{2}: V_{\widetilde{K}}\left(w_{1}, w_{2}\right) \leq \log 1 / \rho\right\}
$$

must hit the complex line $\left\{\left(w_{1}, w_{2}\right) \in \mathbf{C}^{2}: w_{1}=s\right\}$. Thus $f$ cannot be extended holomorphically beyond $E_{1 / \rho}$; i.e., $1 / \rho \geq 1 / R$ or $\rho \leq R$. Since we always have $R \leq \rho$, equality holds.

In the standard $z=\left(z_{1}, z_{2}\right)$ coordinates, a variety $L=L_{q}$ as in Theorem 2.4 through a point $q \in \mathbf{C}^{2} \backslash K$ is of the form

$z=\left(z_{1}, z_{2}\right)=f(t)=\left(f_{1}(t), f_{2}(t)\right)=\left(c_{1}+t b_{1}+\bar{b}_{1} / t, c_{2}+t b_{2}+\bar{b}_{2} / t\right),|t| \geq 1$

where $\mathbf{b}=\left(b_{1}, b_{2}\right) \in \mathbf{C}^{2}, \mathbf{c}=\left(c_{1}, c_{2}\right) \in \mathbf{R}^{2}$, and $V_{K}(f(t))=\log |t|$; the set $f(T)$ is an ellipse $\mathcal{E} \subset \mathbf{R}^{2}$ (possibly degenerate). In the $\left(w_{1}, w_{2}\right)=\left(z_{1}+i z_{2}, z_{1}-i z_{2}\right)$ coordinates, the parameterization of $S(L)$ has the form

$$
\left(w_{1}, w_{2}\right)=h(t)=\left(h_{1}(t), h_{2}(t)\right)=\left(f_{1}(t)+i f_{2}(t), f_{1}(t)-i f_{2}(t)\right) .
$$

Since $V_{\widetilde{K}}\left(w_{1}, w_{2}\right)=V_{K}\left(z_{1}, z_{2}\right)($ see $(3.1))$, we have, in particular, $V_{\widetilde{K}}(h(t))=$ $\log |t|$. Direct calculation shows

$$
h_{1}(t)=\alpha+\beta t+\gamma / t \text { and } h_{2}(t)=\bar{\alpha}+\bar{\gamma} t+\bar{\beta} / t
$$

where $\alpha=c_{1}+i c_{2}, \beta=b_{1}+i b_{2}$, and $\gamma=\bar{b}_{1}+i \bar{b}_{2}$. As in (2.9), the formulas in (3.9) define a holomorphic map $h=\left(h_{1}, h_{2}\right)$ from $\mathbf{C} \backslash\{0\}$ into $\mathbf{C}^{2}$ where 
$h(\mathbf{C} \backslash\{0\})=S(L) \cup S\left(L^{*}\right)$. Note that the conjugate leaf $L^{*}$ given by $t \mapsto \mathbf{c}+t \overline{\mathbf{b}}+\mathbf{b} / t$ transforms in $\left(w_{1}, w_{2}\right)$-coordinates to $S\left(L^{*}\right)$ given by

$$
\tilde{h}_{1}(t):=\alpha+\gamma t+\beta / t, \quad \tilde{h}_{2}(t):=\bar{\alpha}+\bar{\beta} t+\bar{\gamma} / t .
$$

We consider leaves $S(L)=S\left(L_{q}\right)$ where $S(q)=(s, w) \in \mathbf{C}^{2}$ satisfies $h_{K}(s)=$ $V_{\widetilde{K}}(s, w)$. Before giving the proof of the "only if" direction of Theorem 3.1, we need a lemma which shows that if $g_{K}=h_{K}$, then such leaves, for $|s|$ sufficiently large, project conformally in the $w_{1}$ variable.

Lemma 3.5. Suppose $g_{K}=h_{K}$. There exists $R^{\prime}>0$ such that for all $|s|>$ $R^{\prime}$, if $(s, w) \in \mathbf{C}^{2}$ satisfies $h_{K}(s)=V_{\widetilde{K}}(s, w)$, then the parametrization $t \mapsto$ $\left(h_{1}(t), h_{2}(t)\right)$ of $S(L)$ has the property that $h_{1}$ is conformal on $\mathbf{C} \backslash \bar{\Delta}$.

Proof. We will use the fact that

(*) a rational map $\psi: \mathbf{C} \backslash \bar{\Delta} \rightarrow \mathbf{C}$ of the form $\psi(t)=a t+b / t$ with $|b| \leq|a|$ is a conformal map on $\mathbf{C} \backslash \bar{\Delta}$.

First of all, we show that there exists $M>0$ such that for all $|s|>1$,

$$
h_{K}(s)=\inf _{\left|w_{2}\right|<M|s|} V_{\widetilde{K}}\left(s, w_{2}\right) .
$$

For, since $V_{\widetilde{K}} \in L^{+}\left(\mathbf{C}^{2}\right)$, there exists $C_{1}$ such that

$$
V_{\widetilde{K}}(s, w) \geq \log ^{+}|(s, w)|+C_{1}=\frac{1}{2} \log ^{+}\left(|s|^{2}+|w|^{2}\right)+C_{1} ;
$$

since $h_{K}=g_{K} \in L(\mathbf{C})$, there exists $C_{2}$ such that

$$
g_{K}(s)<\log ^{+}|s|+C_{2} .
$$

We may assume $C_{2}>C_{1}$. For $|s|>1$, we solve for $w$ in the inequality

$$
\frac{1}{2} \log \left(|s|^{2}+|w|^{2}\right)+C_{1} \geq \log |s|+C_{2},
$$

to obtain $|w| \geq|s| \sqrt{e^{2\left(C_{2}-C_{1}\right)}-1}$. We can take $M=\sqrt{e^{2\left(C_{2}-C_{1}\right)}-1}$.

Next, we show there exist numbers $R, R^{\prime}>0$ such that for all $(s, w) \in \mathbf{C}^{2}$ satisfying $|s|>R^{\prime}$ and $|w|<M|s|$, a leaf $S(L)$ through $(s, w)$ parametrized as in (3.9) satisfies $|\gamma| /|\beta|<R$ with $(s, w)=h(t)$ for some $|t|>R$. To see this, note first that the leaf parameter $t$ grows uniformly with $s$ :

$$
\log |t|=V_{\widetilde{K}}(s, w) \geq \log |(s, w)|+C_{1} \geq \log |s|+C_{1} \text { so that }|t| \geq|s| e^{C_{1}} .
$$

Now $h(\partial \Delta) \subset \widetilde{K}$ implies that in the $\left(z_{1}, z_{2}\right)$-coordinates, $\left(c_{1}, c_{2}\right) \in K$. Thus for any leaf, $|\alpha|=\left|c_{1}+i c_{2}\right| \leq C_{3}=C_{3}(K)$ is uniformly bounded since $K$ is compact. Consider $(s, w)$ such that $|w|<M|s|$. For any $\alpha$ with $|\alpha| \leq C_{3}$ we have the estimates

$$
\begin{aligned}
|w-\bar{\alpha}| \leq & |w|+|\alpha|<M|s|+|\alpha| \leq M|s-\alpha|+(M+1)|\alpha| \leq M|s-\alpha| \\
& +(M+1) C_{3} .
\end{aligned}
$$

Thus if $s$ satisfies $|s| \geq \frac{(2 M+1) C_{3}}{M},(3.13)$ implies that $|w-\bar{\alpha}|<2 M|s-\alpha|$. 
Given $(s, w)$ with $|s| \geq \frac{(2 M+1) C_{3}}{M}$ and $|w|<M|s|$, consider a leaf $S(L)$ through $(s, w)$ parametrized as in (3.9). For $s=h_{1}(t), w=h_{2}(t)$, using (3.9) and the fact that $|\alpha| \leq C_{3}$,

$$
|\bar{\gamma} t+\bar{\beta} / t|=|w-\bar{\alpha}|<2 M|s-\alpha|=2 M|\beta t+\gamma / t|
$$

which gives

$$
\frac{|\gamma|}{|\beta|}<\frac{2 M|t|^{2}+1}{|t|^{2}-2 M}=: \phi(t) .
$$

As $t \rightarrow \infty$, clearly $\phi(t) \rightarrow 2 M$. Thus there exists $R_{1}>0$ such that if $|t|>R_{1}$, then $\phi(t)<3 M$. Finally, take $R^{\prime}>\max \left\{R_{1} e^{-C_{1}}, \frac{(2 M+1) C_{3}}{M}, 3 M e^{-C_{1}}\right\}$. Then for all $(s, w)$ with $|s|>R^{\prime}$ and $|w|<M|s|$, (3.12) implies that $s=h_{1}(t)$ for some $|t| \geq R^{\prime} e^{C_{1}}=: R$, and by the choice of $R^{\prime}$ we have $R>R_{1}$, so that $|\gamma| /|\beta|<3 M<R$.

Now with $M>0$ so that (3.11) holds, and $R, R^{\prime}$ as in the previous paragraph, we fix $s$ with $|s|>R^{\prime}$. Pick $(s, w) \in \mathbf{C}^{2}$ such that $h_{K}(s)=V_{\widetilde{K}}(s, w)$ and consider the leaf $S(L)$ through $(s, w)$ parametrized as in (3.9). To show that $h_{1}$ is conformal, from (*) it suffices to show that $|\beta| \geq|\gamma|$. Suppose $|\beta|<|\gamma|$. We have $s=h_{1}(t)$ for some $t$ with $|\gamma| /|\beta|<R<|t|$, from which it follows that $|\beta||t| /|\gamma|>1$. Now

$$
s=\alpha+\beta t+\frac{\gamma}{t}=\alpha+\gamma\left(\frac{\beta t}{\gamma}\right)+\frac{\beta}{(\beta t / \gamma)}=: \alpha+\gamma t^{\prime}+\frac{\beta}{t^{\prime}},
$$

i.e., the plane $\left\{w_{1}=s\right\}$ intersects the conjugate leaf $S\left(L^{*}\right)$ at a point $\left(s, w^{\prime}\right)$ corresponding to the parameter $t^{\prime}=\beta t / \gamma \in \mathbf{C} \backslash \bar{\Delta}$ (see (3.10)). Then

$$
h_{K}(s) \leq V_{\widetilde{K}}\left(s, w^{\prime}\right)=\log \left|t^{\prime}\right|=\log \frac{|\beta|}{|\gamma|}|t|<\log |t|=V_{\widetilde{K}}(s, w),
$$

which contradicts the fact that $h_{K}(s)=V_{\widetilde{K}}(s, w)$.

Proof of "only if" in Theorem 3.1. We show that if $h_{K}=g_{K}$ on $\mathbf{C} \backslash K$, then $\partial K$ is an ellipse. Fix $s \in \mathbf{C} \backslash K$ with $|s|>R^{\prime}$ where $R^{\prime}$ is as in Lemma 3.5. Choose $w \in \mathbf{C}$ with $h_{K}(s)=V_{\widetilde{K}}(s, w)$. Let $S(L)$ be a variety in $\mathbf{C}^{2} \backslash \widetilde{K}$ containing $(s, w)$ parametrized in $\left(w_{1}, w_{2}\right)$-coordinates by $h(t)=\left(h_{1}(t), h_{2}(t)\right)$ as in (3.9). Lemma 3.5 shows that $h_{1}$ is a conformal map of $\mathbf{C} \backslash \bar{\Delta}$ onto its image $h_{1}(\mathbf{C} \backslash \bar{\Delta}):=\mathbf{C} \backslash \bar{U}$. Moreover, $\bar{U} \subset K ; h_{1}(T)=\partial U$ is an ellipse or a line segment; and $V_{\widetilde{K}}(h(t))=\log |t|$ for $|t| \geq 1$. Now $(s, w)=h\left(t_{0}\right)$ for some $t_{0} \in \mathbf{C} \backslash \bar{\Delta}$. Thus $h_{1}\left(t_{0}\right)=s$, and, using the conformality of $h_{1}$,

$$
g_{K}(s)=h_{K}(s)=V_{\widetilde{K}}(s, w)=\log \left|t_{0}\right|=g_{\bar{\Delta}}\left(t_{0}\right)=g_{\bar{U}}(s) .
$$

But $\bar{U} \subset K$ implies that $g_{K} \leq g_{\bar{U}}$; both functions are harmonic in $\mathbf{C} \backslash K$; thus, equality at one point $s \in \mathbf{C} \backslash K$ implies equality throughout. Now $K$ and $\bar{U}$ are compact, convex sets in $\mathbf{C}$ with $g_{K}=0$ on $K$ and $g_{\bar{U}}=0$ on $\bar{U}$; hence $K=\bar{U}$. Since $K$ has nonempty interior, $\partial K=\partial U$ is an ellipse.

Remark. There is no known Bernstein-Walsh type theorem for harmonic functions in $\mathbf{R}^{N}$ when $N>2$ (but see [BL]); thus it is not clear, apriori, whether the "only if" direction of Theorem 3.1 remains valid in this case. 


\section{The function $V_{K}^{(1)}$}

Following [BCL], let $K \subset \mathbf{C}^{N}$ be a regular compact set and let $p_{d}$ be a polynomial of degree $d$. Then $p_{d}(K)$ is a regular compact set in $\mathbf{C}$ and $\frac{1}{d} V_{p_{d}(K)}\left(p_{d}(z)\right) \leq$ $V_{K}(z)$. Conversely, if $\left\|p_{d}\right\|_{K} \leq 1$, then $V_{p_{d}(K)}(w) \geq \log ^{+}|w|$ for all $w \in \mathbf{C}$; in particular, $V_{p_{d}(K)}\left(p_{d}(z)\right) \geq \log ^{+}\left|p_{d}(z)\right|$, from which it follows that $V_{K}(z) \leq$ $\sup _{p_{d}} \frac{1}{d} V_{p_{d}(K)}\left(p_{d}(z)\right)$; i.e.,

$$
V_{K}(z)=\sup _{p_{d}} \frac{1}{d} V_{p_{d}(K)}\left(p_{d}(z)\right) .
$$

Using only the polynomials of degree one, we define

$$
V_{K}^{(1)}(z):=\sup \left\{V_{\ell(K)}(\ell(z)): \ell \text { is complex affine }\left(\ell: \mathbf{C}^{N} \rightarrow \mathbf{C}\right)\right\} .
$$

From the work of Baran and Lundin [Ba1], [Ba2], [L1], it follows that $V_{K}^{(1)}=V_{K}$ for $K \subset \mathbf{R}^{N}$ a compact, convex body which is symmetric with respect to the origin. It was shown in [BCL] that for the simplex $S_{2}:=\left\{\left(x_{1}, x_{2}\right) \in \mathbf{R}^{2}: x_{1}, x_{2} \geq\right.$ $\left.0 ; x_{1}+x_{2} \leq 1\right\}$ in $\mathbf{R}^{2}, V_{S_{2}}^{(1)} \neq V_{S_{2}}$. Indeed, more is true. Before we proceed, we mention two results from [BLM] which we will need. Here, $K, K^{\prime} \subset \mathbf{C}^{N}$ are compact and regular.

(i) $\left\|V_{K}^{(1)}-V_{K^{\prime}}^{(1)}\right\|_{\mathbf{C}^{N}} \leq\left\|V_{K}-V_{K^{\prime}}\right\|_{\mathbf{C}^{N}}$ (Lemma 3.3, [BLM]).

(ii) $V_{K}^{(1)}$ is continuous. (Proposition 3.5, [BLM]).

Using the fact that $\ell(K)$ is regular, and observing that in the definition (0.2) of $V_{K}^{(1)}$ we need only utilize $\ell(z)=<a, z>$ with $|a|=1$, it follows easily that for each $z \in \mathbf{C}^{N}$, there exists $\ell(z)=<a, z>$ with $|a|=1$ for which $V_{K}^{(1)}(z)=V_{\ell(K)}(\ell(z))$ (cf., Proposition 2.14 [Ma2]).

Let $\Pi$ denote the collection of regular compact sets $K$ in $\mathbf{C}^{N}$ that are polynomially convex; i.e.,

$$
\hat{K}:=\left\{z \in \mathbf{C}^{N}:|p(z)| \leq\|p\|_{K} \text { for all holomorphic polynomials } p\right\}=K .
$$

Klimek has shown [K2] that

$$
\Gamma(E, F):=\left\|V_{E}-V_{F}\right\|_{\mathbf{C}^{N}}, \quad E, F \in \Pi
$$

defines a metric $\Gamma$ on $\Pi$. Now it is straightforward to show (cf., Prop. 4.2 [BCL]) that if $K \in \Pi$ satisfies $V_{K}^{(1)}=V_{K}$, then $K$ is lineally convex; i.e., the complement of $K$ is the union of complex hyperplanes. Let $\Pi^{1}$ denote the collection of lineally convex, regular compact sets $K$ in $\mathbf{C}^{N}$ with the property that $\ell(K)$ is polynomially convex in $\mathbf{C}$ for each $\ell(z)=\langle a, z>$. It follows from [Ma], Chapter 3 or [Ma2], Lemma 2.4 that such sets $K$ are polynomially convex in $\mathbf{C}^{N}$; i.e., $\Pi^{1} \subset \Pi$. Define

$$
\Gamma^{(1)}(E, F):=\left\|V_{E}^{(1)}-V_{F}^{(1)}\right\|_{\mathbf{C}^{N}}, \quad E, F \in \Pi^{1} .
$$

Then $\Gamma^{(1)}$ defines a metric on $\Pi^{1}$ (Proposition 3.7 [Ma] or Proposition 2.10 [Ma2]); moreover, from (i),

$$
\Gamma^{(1)}(E, F) \leq \Gamma(E, F) \text {. }
$$


The set $\theta:=\left\{K \in \Pi^{1}: V_{K}^{(1)} \neq V_{K}\right\}$ is a non-empty open set in $\Pi^{1}$ equipped with the $\Gamma^{(1)}$ metric (this is essentially Proposition 6.1 of [BCL] together with (ii)). Moreover, if we let $\mathcal{R}$ be the collection of compact convex bodies in $\mathbf{R}^{2}$ - note that $\mathcal{R} \subset \Pi^{1}-$ then Corollary 6.2 of [BCL] states that

$$
\theta_{\mathcal{R}}:=\left\{K \in \mathcal{R}: V_{K}^{(1)} \neq V_{K}\right\}
$$

is a non-empty open set in $\mathcal{R}$ equipped with the $\Gamma^{(1)}$ metric. In Corollary 4.2 we show that $\theta_{\mathcal{R}}$ is dense in $\mathcal{R}$ with respect to both $\Gamma$ and $\Gamma^{(1)}$. The key ingredient is a strengthening and generalization of the simplex result. To state this, recall in Theorem 2.4 we showed that if $K \subset \mathbf{R}^{N}$ is a convex body, then through any point $q \in \mathbf{C}^{N} \backslash K$ there is a one-dimensional variety $L_{q}=F(\mathbf{C} \backslash \bar{\Delta})$ where $F=\left(F_{1}, \ldots, F_{N}\right)$ with $F_{n}(t)=a_{n 0}+a_{n 1} t+\bar{a}_{n 1} / t$ for some $a_{n 0} \in \mathbf{R}$ and $a_{n 1} \in \mathbf{C}$; $F(T) \subset K$; and $V_{K}(F(t))=\log |t|$ for $t \in \mathbf{C} \backslash \Delta$. These sets $L_{q}$ are complex ellipses; however, if the coefficients $a_{n 1}$ are real, then $L_{q}$ is a complex line. For example, in $\mathbf{C}^{2}$, such an $L_{q}$ is the complex line $a_{21}\left(z_{1}-a_{10}\right)=a_{11}\left(z_{2}-a_{20}\right)$. We call such an $L_{q}$ degenerate.

Proposition 4.1. If $K$ is a convex polygon in $\mathbf{R}^{2}$ having no two sides parallel, then $V_{K}^{(1)}(z)<V_{K}(z)$ at all points $z \in \mathbf{C}^{2} \backslash \mathcal{L}$ where $\mathcal{L}$ is the union of all degenerate $L_{q}$.

Remark 1. In the case of the simplex $S_{2}$, direct calculation (cf., [Ma]) shows that $\mathcal{L}$ is the union of the three families of complex lines whose intersection with $\mathbf{R}^{2}$ are real lines through one of the vertices of $S_{2}$. This is a three (real) dimensional set in $\mathbf{C}^{2}$. Thus, in some sense, $V_{S_{2}}^{(1)}<V_{S_{2}}$ on "most" of $\mathbf{C}^{2}$. In general, since $L_{q} \cap K$ must be a line segment for a degenerate $L_{q}$ and a convex polygon $K$, the set $\mathcal{L}$ is certainly contained in the set $\mathcal{L}^{\prime}$ of all complex lines whose intersections with $\mathbf{R}^{2}$ are real lines which intersect $K$. Thus, for example, all points of the form $(R, i)$ with $R>\max _{\left(x_{1}, x_{2}\right) \in K}\left|x_{1}\right|$ lie in $\mathbf{C}^{2} \backslash \mathcal{L}^{\prime}$, and hence in $\mathbf{C}^{2} \backslash \mathcal{L}$. In particular, $\left\{z: V_{K}^{(1)}(z)<V_{K}(z)\right\}$ is nonempty and unbounded.

Remark 2. The assumption that no two sides of $K$ are parallel is essential. For the unit square $K=[-1,1] \times[-1,1], V_{K}^{(1)}=V_{K}$; indeed, this is true for any convex polygon that is symmetric with respect to the origin.

Proof. For $z \notin \mathcal{L}$, by Theorem 2.4, we take a variety $L_{z}=f(\mathbf{C} \backslash \bar{\Delta})$ through $z$ for which $V_{K}(f(t))=\log |t|$. For simplicity, we write $f(t)=\left(\left(c_{1} t+\bar{c}_{1} / t\right),\left(c_{2} t+\right.\right.$ $\left.\bar{c}_{2} / t\right)$ ). Since $z \in \mathbf{C}^{2} \backslash \mathcal{L}, L_{z}$ is nondegenerate and $L_{z} \cap \mathbf{R}^{2}$ is a real (nondegenerate) ellipse forming the boundary in $\mathbf{R}^{2}$ of a convex, compact set $U \subset K$ with nonempty interior. In particular, $U$ contains no vertices of $K$.

Since $K$ is regular, we can find a linear map $\ell: \mathbf{C}^{2} \rightarrow \mathbf{C}$ such that $V_{K}^{(1)}(z)=$ $V_{\ell(K)}(\ell(z))$. Then $\ell \circ f$ is a holomorphic map from $\mathbf{C} \backslash \bar{\Delta}$ to $\mathbf{C}$ which is continuous up to $T=\partial \Delta$, and $\ell \circ f(T)=\ell(\partial U)$ where $\ell(U) \subset \ell(K)$. Note that replacing $t$ by $e^{i \theta} t$ in the parameterization for $f$ still gives the same variety $L_{z}$. Moreover, for the conjugate leaf $L_{z}^{*}, L_{z}^{*} \cap \mathbf{R}^{2}=L_{z} \cap \mathbf{R}^{2}$. Hence we may normalize our parameters 
as follows. If $\ell(z)=a z_{1}+b z_{2}$ with $\Im(a \bar{b}) \geq 0$, we may assume that $c_{1} \in \mathbf{R}^{+}$, and $\Im\left(c_{2}\right) \leq 0$. Under this normalization, we will show that $\ell \circ f$ is $1-1$. We have

$$
\ell \circ f(t)=\left(a c_{1}+b c_{2}\right) t+\left(a \bar{c}_{1}+b \bar{c}_{2}\right) \frac{1}{t}
$$

and it suffices to show (cf., (*) in the proof of Lemma 3.5) that $\left|a c_{1}+b c_{2}\right| \geq$ $\left|a \bar{c}_{1}+b \bar{c}_{2}\right|$. We compute

$$
\begin{aligned}
& \left|a c_{1}+b c_{2}\right|^{2}=\left|a c_{1}\right|^{2}+\left|b c_{2}\right|^{2}+2 c_{1}\left[\Re(a \bar{b}) \Re\left(c_{2}\right)-\Im\left(c_{2}\right) \Im(\bar{a} b)\right] \\
& \left|a \bar{c}_{1}+b \bar{c}_{2}\right|^{2}=\left|a c_{1}\right|^{2}+\left|b c_{2}\right|^{2}+2 c_{1}\left[\Re(a \bar{b}) \Re\left(c_{2}\right)+\Im\left(c_{2}\right) \Im(\bar{a} b)\right] .
\end{aligned}
$$

If $\Im(\bar{a} b)<0$ we normalize so that $\Im\left(c_{2}\right) \geq 0$ and repeat the above procedure. Thus $\ell \circ f$ is a one-to-one conformal map of $\mathbf{C} \backslash \bar{\Delta}$ onto $\mathbf{C} \backslash \ell(U)$. Writing $z=f(t)$ we have

$$
V_{K}(z)=\log |t|=V_{(\ell \circ f)(T)}((\ell \circ f)(t))=V_{\ell(\partial U)}(\ell(z))=V_{\ell(U)}(\ell(z)) .
$$

Now $\ell(U) \subset \ell(K)$, and in general, $\ell(U)$ is the region bounded by an ellipse while $\ell(K)$ is the region bounded by a convex polygon. In the case where $\ell$, considered as a real-linear map from $\mathbf{R}^{2}$ to $\mathbf{R}^{2}$, has rank two, $\ell(K)$ is a nondegenerate convex polygon (i.e., with nonempty interior in $\mathbf{R}^{2}$ ), and $\ell(U)$ is the region bounded by a nondegenerate ellipse. Since $\ell(U)$ thus contains no vertices of $\ell(K)$, $\ell(K) \backslash \ell(U)$ has positive area.

The case where $\ell(K)$ is degenerate (i.e., a line segment) occurs when $\ell$, considered as a real-linear map from $\mathbf{R}^{2}$ to $\mathbf{R}^{2}$, has rank one. In this case we may consider $\ell$ as a projection map $\ell: \mathbf{R}^{2} \rightarrow \mathbf{R}$. Suppose that $\ell(K)=[\alpha, \beta]$. This means that $\alpha$ and $\beta$ must be the projections under $\ell$ of parallel supporting lines $M_{1}$ and $M_{2}$ for $K$. These lines cannot both contain sides of $K$ as no two sides of $K$ are parallel. Thus at least one of the intersections $M_{1} \cap K$ or $M_{2} \cap K$ is a vertex of $K$. If it were the case that $\ell(U)=[\alpha, \beta]$, then at least one of the intersections $M_{1} \cap U$ or $M_{2} \cap U$ is a vertex of $K$. But this cannot happen as $U$ contains no vertices of $K$. Thus $\ell(K) \backslash \ell(U)$ contains a (nontrivial) line segment.

Now $\ell(U)$ and $\ell(K)$ are nonpolar (since each contains a line segment), and polynomially convex since both sets are, in particular, convex. Moreover, in each case described above $\ell(K) \backslash \ell(U)$ is nonpolar. From classical potential theory (see e.g., [R]) we conclude that $V_{\ell(K)}(\ell(z))<V_{\ell(U)}(\ell(z))$. But

$$
V_{K}^{(1)}(z)=V_{\ell(K)}(\ell(z)) \text { and } V_{\ell(U)}(\ell(z))=V_{K}(z),
$$

so the proposition is proved.

Corollary 4.2. The set $\theta_{\mathcal{R}}$ is dense in $\mathcal{R}$ with respect to both $\Gamma$ and $\Gamma^{(1)}$.

Proof. Fix $K \in \mathcal{R}$. Given $\epsilon>0$ we may approximate $K$ from the outside by a convex polygon $P$ such that $\left\|V_{P}-V_{K}\right\|_{\mathbf{C}^{N}}<\epsilon$. By modifying $P$, if necessary, we can assume that no two sides are parallel; hence $V_{P}^{(1)} \neq V_{P}$. Thus $\theta_{\mathcal{R}}$ is dense in $\Gamma$. Since $\Gamma^{(1)}(P, K) \leq \Gamma(P, K), \theta_{\mathcal{R}}$ is dense in $\Gamma^{(1)}$ as well. 


\section{References}

[BL] Bagby, T., Levenberg, N.:Bernstein theorems for harmonic functions. Methods of Approx. Theory in Complex Analysis and Mathematical Physics, Lecture Notes in Math. (Springer-Verlag), 1550, 7-18 (1993)

[Ba1] Baran, M.: Siciak's extremal function of convex sets in $\mathbf{C}^{n}$. Annales Polonici Math. 48, 275-280 (1988)

[Ba2] Baran, M.: Plurisubharmonic extremal functions and complex foliations for the complement of convex sets in $\mathbf{R}^{n}$. Michigan Math. J. 39, 395-404 (1992)

[BK] Bedford, E., Kalka, M.: Foliations and complex Monge-Ampère equations, Comm. Pure Appl. Math. 30(5), 543-571 (1977)

[BLM] Bloom, T., Levenberg, N., Ma'u, S.: Robin functions and extremal functions. Annales Polonici Math. 80, 55-84 (2003)

[Bo] Borell, S.: Pluricomplex Green functions and invariant pseudodistances in convex domains. Mid Sweden University Masters Thesis

[BCL] Bos, L., Calvi, J.-P., Levenberg, N.: On the Siciak extremal function for real compact convex sets in $\mathbf{R}^{N}$. Arkiv for Mat. 39(2), 245-262 (2001)

[K] Klimek, M.: Pluripotential Theory. Oxford University Press, Oxford, 1991

[K2] Klimek, M.: Metrics associated with extremal plurisubharmonic functions. Proc. Amer. Math. Soc. 123, 2763-2770 (1995)

[Le1] Lempert, L.: La métrique de Kobayashi et la représentation des domaines sur la boule. Bull. Soc. Math. France 109(4), 427-474 (1981)

[Le2] Lempert, L.: Symmetries and other transformations of the complex Monge-Ampre equation. Duke Math. J. 52(4), 869-885 (1985)

[Le3] Lempert, L.: Intrinsic distances and holomorphic retracts. Complex analysis and applications '81 (Varna, 1981), 1984, pp. 341-364

[L1] Lundin, M.: The extremal plurisubharmonic function for the complement of convex, symmetric subsets of $\mathbf{R}^{n}$. Michigan Math. J. 32, 197-201 (1985)

[L2] Lundin, M.: Thesis (unpublished)

[Ma] Ma'u, S.: Plurisubharmonic functions of logarithmic growth. PhD thesis, University of Auckland, 2003

[Ma2] Ma'u, S.: Plurisubharmonic functions characterized by one-variable extremal functions. Preprint

[M] Momm, S.: An extremal plurisubharmonic function associated to a convex pluricomplex Green function with pole at infinity. J. Reine Angew. Math. 471, 139-163 (1996)

[ND] Nguyen Thanh Van Djebbar, B.: Propriétés asymptotiques d'une suite orthonormale de polynômes harmoniques. Bull. Sc. Math. 113, 239-251 (1989)

[R] Ransford, T.: Potential Theory in the Complex Plane. Cambridge Univ. Press, Cambridge, 1995

[S] Siciak, J.: Extremal plurisubharmonic functions in $\mathbf{C}^{N}$. Annales Polonici Math. 39, 175-211 (1981)

[W] Walsh, J.L.: Maximal convergence of sequences of harmonic polynomials. Ann. Math. 38, 321-354 (1936) 